\title{
Uncertainty Modeling and Robust Control in Urban Traffic *
}

\author{
T. Tettamanti ${ }^{*}$ I. Varga ${ }^{* *}$ T. Péni ${ }^{* *}$ T. Luspay ${ }^{* *}$ \\ B. Kulcsár ${ }^{* * *}$ \\ * Department of Control and Transport Automation, Budapest \\ University of Technology and Economics, 1111, Budapest, Hungary \\ (e-mail: tettamanti@mail.bme.hu) \\ ** Computer and Automation Research Institute, Hungarian Academy \\ of Sciences, 1111, Budapest, Hungary (e-mail: \{ivarga, \\ pt\}@scl.sztaki.hu) \\ *** Department of Signals and Systems, Chalmers University of \\ Technology, SE-412 96, Gothenburg, Sweden (e-mail: \\ kulcsar@chalmers.se)
}

\begin{abstract}
The paper investigates the problem of uncertainty modeling and constrained robust control of urban traffic. Linear polytopic approach is used by state-space representations to describe the uncertain network system. In order to handle model mismatches, robust and infinite horizon model predictive control (MPC) method is proposed. The control strategy is an efficient method to reduce travel time and improve homogeneous traffic flow under changing model conditions. Centralized numerical solution has been carried out as a solution of Linear Matrix Inequalities (LMI) by using semidefinite programming (SDP). Closed-loop control results were tested in simulation environment taking alternative model uncertainty levels into account.
\end{abstract}

Keywords: urban traffic control, robust, minimax, uncertainty

\section{INTRODUCTION}

During the past decades, several results have been published in the field of model control of urban road traffic network. Nevertheless, the methodologies applied for optimal control have not taken any uncertainties into consideration. Considering the urban traffic control problem from the perspective of modern control theory a commonly used modeling paradigm can be found. The Store-and-forward model of urban network (Gazis and Potts, 1963) is easily transformable into linear time invariant (LTI) state space form eligible already for several control techniques. Just to mention some recent papers based on this model we refer to Tettamanti et al. (2008), Aboudolas et al. (2009) and de Oliveira and Camponogara (2010). These publications present detailed methods for optimal traffic control. At the same time, they do not concern the robust property of the control system. Plant model uncertainties are always neglected. To confront this problem we introduce an alternative technique based on the minimax MPC using (LMIs) presented in Kothare et al. (1996). To solve LMI based optimization process we apply the SDP algorithm

\footnotetext{
* The work is connected to the scientific program of the "Development of quality-oriented and harmonized $\mathrm{R}+\mathrm{D}+\mathrm{I}$ strategy and functional model at BME" project. This project is supported by the New Hungary Development Plan (Project ID: TÁMOP-4.2.1/B09/1/KMR-2010-0002) and by the Hungarian Scientific Research Fund (OTKA) through grant No. CNK 78168 and by János Bólyai Research Scholarship of the Hungarian Academy of Sciences which are gratefully acknowledged. This work has been supported by Chalmers' new initiatives in Transportation, therefore B. Kulcsár acknowledges the support of the Area of Advance in Transportation.
}

implemented in the simulation environment. The proposed controller deals only with signal split optimization. Offsets and optimal cycle time are assumed to be fixed or calculated by other algorithms.

The first part of the paper consist the detailed analysis of the state space model of urban traffic based on the well known Store-and-forward approach. A centering technique of the system is also presented. Furthermore, the polytopic approach is introduced to describe the state uncertainties. In the second part, a minimax control approach is discussed as a potential robust method for dealing with uncertainties. We show how to involve LMI based control in urban traffic network management with the appropriate handling of constraints. Finally, the simulation results are presented for proving the robust property of the system which is able to attenuate state uncertainties and hereby to improve network efficiency, subjected to control input and output constraints.

\section{URBAN TRAFFIC MODELING}

\subsection{From Store-and-forward traffic modeling to state space} representation

The traffic modeling has two main approaches: micro and macro modeling techniques. Micro models consider each vehicle in the traffic process as individual object with several individual properties (e.g. prespecified driver behavior, vehicle parameters). Therefore, the micro modeling is usable mostly for traffic simulations. Macro modeling approach does not specify much details of the vehicles. 
It mostly deals with the traffic flows on the whole. Due to its simplicity, macro modeling is a practical approach in real-word applications and suitable for control processes. A common macro modeling approach for urban road traffic is based on the Store-and-forward dynamic model Gazis and Potts (1963) which represents a simple vehicle-conservation equation between two neighbouring intersections $(M, N)$.

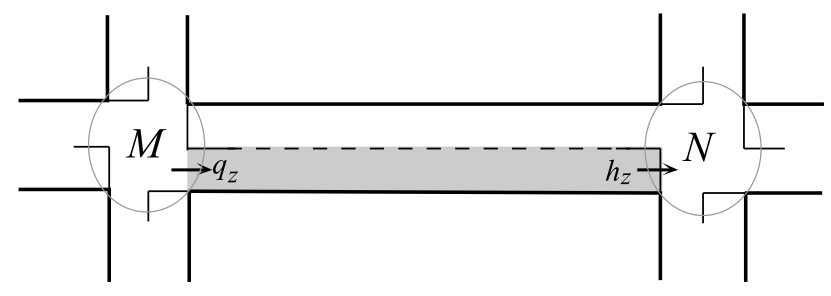

Fig. 1. Generalized urban road link

Considering Fig. 1 the traffic dynamics is described by the following linear equation:

$$
x_{z}(k+1)=x_{z}(k)+T\left[q_{z}(k)-h_{z}(k)\right]
$$

where $x_{z}$ is the number of vehicles expressed in Passenger Car Equivalent (PCE), $q_{z}$ the inflow, $h_{z}$ the outflow $(P C E / h)$ of link $z$ during $[k T,(k+1) T]$ where $k$ is the discrete time step and $T$ the sampling time. It has to be emphasized that the traffic flows generated and consumed by the link are neglected for simplicity. These flows can be produced by parking lots or uncontrolled intersections. If they are known parameters they can be easily added to the model (1). Nevertheless, the paper's aim is to deal with them as bounded state uncertainties which will be presented in the following sections.

Equation (1) gives a single link dynamics. In order to obtain network dynamics each link equation is needed. The network can be represented by directed graphs composed of nodes and arcs. The nodes $j \in J$ denote the controlled intersections and the arcs $z \in Z$ the links. In this case the meaning of $q_{z}$ and $h_{z}$ has to be given. Equation 1 is redefined depending of the network dynamics:

$$
\begin{aligned}
& x_{z}(k+1)=x_{z}(k)+ \\
& \frac{T}{C}\left[\sum_{w \in I_{M}} \alpha_{w, z} S_{w} \sum_{i \in v_{w}} u_{M, i}(k)-S_{z} \sum_{i \in v_{z}} u_{N, i}(k)\right]
\end{aligned}
$$

$C$ is the cycle time and we assume $T=C . \alpha_{w, z}$ with $w \in I_{M}$ are the turning rates of the links entering junction $M$ towards link $z . S_{w}$ and $S_{z}$ are the saturation rates giving the traffic flow ratio during the green times. The values of $\alpha$ and $S$ are considered known and constant. Nevertheless, they can be measured or estimated (Kulcsár et al., 2005). $\sum_{i \in v_{w}} u_{M, i}$ and $\sum_{i \in v_{z}} u_{N, i}$ represent the green times of intersection $M$ and $N$, respectively (during the cycle time). Equation 2 can be written in matrix form which yields the general LTI state space representation Diakaki et al. (1999):

$$
\begin{aligned}
& x(k+1)=A x(k)+B u(k)+E d(k), \\
& y(k)=C x(k),
\end{aligned}
$$

In our case the state matrix $A$ is practically considered as an identity matrix. The elements of the state vector $x$ represent the number of vehicles of each controlled link. The number of states is equal to the number of controlled links in the network. Matrix $B$ can be constructed by the appropriate allocation of the combinations of saturation and turning rates. The diagonal values of $B$ are negative $S_{z}$ as the product $S_{z} \sum_{i \in v_{z}} u_{N, i}$ represents the outflow of link $z$. The third term $E d(k)$ of (3) represents an additive disturbance where $E=I$. $d$ contains the traffic intending to enter the network at the boundary. Traffic $d$ is considered as a measurable value. Matrix $C$ is an identity matrix as all outputs inside the network are at the same time measured states. The values of $y(k)=x(k)$ are not directly measurable but may be estimated by using Kalman-filter Welch and Bishop (1995), for example. A possible realization for state estimation was published in the paper of Vigos et al. (2007).

The traffic model derived above is valid only with constraints. The states are subject to physical constraints as the maximum number of vehicles $\left(x_{z, \max }\right)$ is defined by the length of $z^{\text {th }}$ link between two junctions (considering PCEs):

$$
0 \leq x_{z}(k) \leq x_{z, \max }
$$

Also the control input is restricted by constraints. On the one hand, $u$ is constrained by the interval of green time:

$$
u_{z, \min } \leq u_{z}(k) \leq u_{z, \max }\left(x_{z}\right)
$$

$u_{z, \max }$ is state dependent since the number of vehicles on the link determines the maximum green time. This constraint assures the positivity of the states. Depending on the system setting $u_{z, \min }$ (for lack of vehicles on a link given) can be zero, which means permanent red signal for the stage in the next control interval. On the other hand, control input $u$ is restricted by the linear combination constraints of green times of junction $j$. The sum of the green times has to be less than $T_{j, \max }$ :

$$
\sum_{z=1}^{O_{j}} u_{z}(k) \leq T_{j, \max }, \quad j=1,2, \ldots, J
$$

where $O_{j}$ is the number of stages at junction $j, T_{j, \max }=$ $T-L_{j}\left(L_{j}\right.$ is the fixed lost time resulted from the geometry of junction $j$ ), and $J$ is the number of controlled intersections.

\subsection{Nominal traffic model}

The traffic model (3) derived from Store-and-forward approach contains an additional term, $E d(k)$. This term cannot be directly handled by the control techniques (neither the later introduced minimax MPC) which are based on the minimization of the general LQ cost functional. Therefore a centered traffic model is needed, where $E d(k)$ can be eliminated (Diakaki et al., 1999). The control is based on a fixed nominal signal plan. First, we have to introduce the following equations which are held for the original model (3): 


$$
\Delta x=x-x^{N}, \Delta u=u-u^{N}, \Delta d=d-d^{N},
$$

where index $N$ denotes the nominal value and $\Delta$ represents the deviation compared to the nominal value. It has to be noted that disturbance $d$ represents exclusively the inflow demand at the boundary of the network (see Sec. 2.1). It is assumed that $d=d^{N}$ and hence, $\Delta d=0$. Furthermore, we assume that in stationary case the state equation is $x^{N}(k+1)=x^{N}(k)$ and thus $x^{N}(k)=x^{N}(k)+B u^{N}(k)+$ $d^{N}(k)$ (with $A=E=I$ ) which result in $B u^{N}+d^{N}=0$. Therefore, we arrive at the eligible state space model without any additive term:

$$
\Delta x(k+1)=A \Delta x(k)+B \Delta u(k)
$$

which implies that our control is designed to find control deviation $\Delta u(k)$. To apply expression (8) an appropriate nominal signal plan is required. Hereunder we introduce a possible method to calculate the nominal control input $u^{N}$. Knowing demands $d^{N}$, it is straightforward to find $u^{N}$ solving the following feasibility problem:

$$
\begin{array}{ll}
\text { find } & u^{N} \\
\text { s.t. } & B u^{N}+d^{N}=0 \\
& u_{z, \min } \leq u_{z}^{N} \leq u_{z, \max } \\
& \sum_{z=1}^{O_{j}} u_{z}^{N} \leq T_{j, \text { max }}
\end{array}
$$

\subsection{Uncertainty modeling in urban traffic}

In the previous parts the general LTI state space representation of urban traffic system was discussed in details. The possible state uncertainties were neglected or considered as known parameters, e.g. in the paper of Diakaki et al. (1999) the demand and exit flows are known values within the link. Typically, state uncertainties appear due to unexpected traffic fluctuations caused by parking places along the road or non-controlled junctions in the network (Fig. 2). The measurements of these disturbing flows would lead to enormous costs in urban network. Therefore, it is more reasonable to treat them as bounded uncertainties.

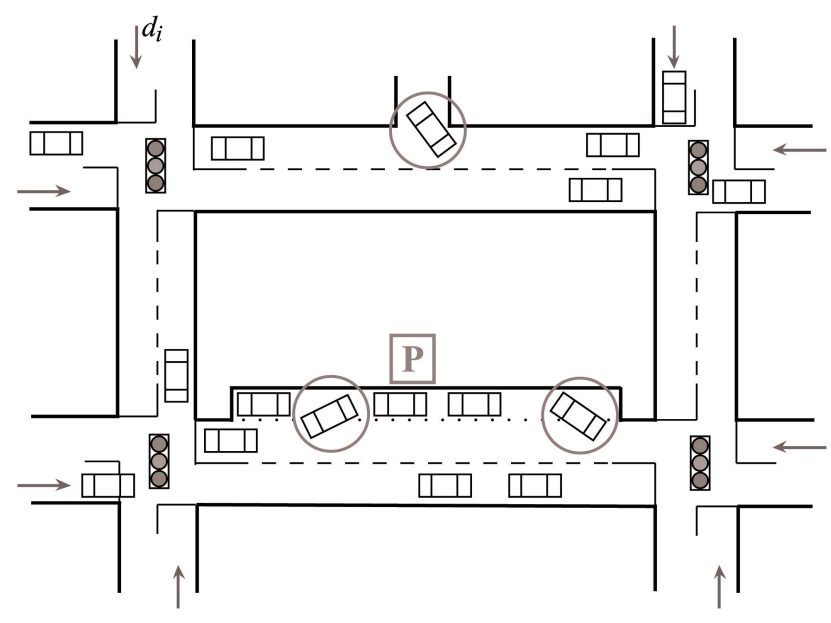

Fig. 2. State uncertainties in urban traffic
A common approach for modeling uncertainties is the use of bounded additive disturbance model. Another potential technique is the multiplicative approach which may involve state uncertainties in the traffic model. In the paper the latter approach was chosen for robust control design.

The uncertainties can be defined on linear time variant (LTV) system matrices $A(k)$ and $B(k)$ within polytope $\Omega$ :

$$
\Omega(k)=\operatorname{Co}\left\{\left[\begin{array}{ll}
A_{1} & B_{1}
\end{array}\right],\left[\begin{array}{ll}
A_{2} & B_{2}
\end{array}\right], \ldots,\left[A_{L} B_{L}\right]\right\}
$$

where $C o$ devotes the convex hull and $L$ is the number of the vertices of uncertainties. Equation (10) expresses that $[A(k) B(k)] \in \Omega$ if for some nonnegative $\lambda_{i}\left(\sum_{i=1}^{L} \lambda_{i}=1\right)$ :

$$
[A(k) B(k)]=\sum_{i=1}^{L} \lambda_{i}\left[A_{i}(k) B_{i}(k)\right]
$$

As we only attend to the state uncertainties throughout this paper, we may conclude that

$$
A(k) \in \Omega=C o\left\{A_{1}(k), A_{2}(k), \ldots, A_{L}(k)\right\}
$$

The vertices of uncertainties can be placed into the current diagonal elements of $A$ as $A=I$ in nominal case. For example, in case of one single uncertain link in the network, polytope $\Omega$ contains the following matrices:

$$
A_{1}=\left[\begin{array}{ccrr}
1 & 0 & \ldots & 0 \\
0 & 1-\alpha_{1} & \vdots \\
\vdots & & \ddots & 0 \\
0 & \ldots & 0 & 1
\end{array}\right], A_{2}=\left[\begin{array}{cccc}
1 & 0 & \ldots & 0 \\
0 & 1 & +\beta_{1} & \vdots \\
\vdots & & \ddots & 0 \\
0 & \ldots & 0 & 1
\end{array}\right]
$$

where $\alpha_{i}$ and $\beta_{i}\left(\alpha_{i}, \beta_{i} \in[0,1]\right)$ represent percent deviations of the measured states. The measure of the uncertainties can be estimated based on prior measurements exclusively. Although $A_{i}(k)$ is time varying, it does not definitely alter in each time step. Therefore, it may be updated in larger time interval (e.g. night hours, morning hours).

In this paper polytopic uncertainty description is considered around the centered representation (8) for control oriented reasons. Authors are aware of the specific structure of the uncertainty, and believes that the before mentioned description is valid if $x(k)>x^{N}(k)$, i.e in oversaturated intersections.

\section{ROBUST CONTROL IN URBAN TRAFFIC NETWORK}

Uncertainties introduced in the previous part can strongly disturb online optimization based traffic control. In the following part an efficient robust traffic control method is introduced to overcome the uncertainty problem.

\subsection{Minimax $M P C$}

The minimax approach is a potential method to deal with uncertainties. An infinite horizon minimax MPC was published in Kothare et al. (1996), which is the basis of our traffic control design. The centered model (8) can be described for state uncertain system as LTV model: 


$$
\begin{aligned}
& \Delta x(k+1)=A(k) \Delta x+B(k) \Delta u(k), \\
& A(k) \in \Omega
\end{aligned}
$$

An efficient robust solution for system (14) is the minimization of a robust performance objective:

$$
\min _{\Delta u(k+i \mid k), i=0,1, \ldots, m-1}\left(\max _{A(k+i) \in \Omega, i \geq 0} J_{p}(k)\right),
$$

where

$$
\begin{aligned}
& J_{p}(k)=\sum_{i=0}^{p}\left(\Delta x(k+i \mid k)^{T} Q_{1} \Delta x(k+i \mid k)+\right. \\
& \left.\Delta u(k+i \mid k)^{T} R \Delta u(k+i \mid k)\right)
\end{aligned}
$$

$Q_{1} \succ 0$ and $R \succ 0$ are symmetric weighting matrices. $p$ is the prediction horizon and $m$ the control horizon. We consider the case of $p=\infty$, which implies the so-called infinite horizon MPC. Finite horizon control laws have weak nominal stability properties (Rawlings and Muske, 1993). To ensure nominal stability of finite horizon MPC one has to impose the terminal state constraint $\Delta x(k+$ $i \mid k)=0(i=m)$ or the contraction mapping principle (Zafiriou, 1990) to tune $Q_{1}, R, m$ and $p$ for stability. These methods, however, cannot guarantee stability in all cases and may imply extreme computational time. Therefore, infinite horizon approach is adopted to guarantee at least nominal stability (Rawlings and Muske, 1993). In the following part instead of the maximization of $A(k+i) \in \Omega, i \geq 0 J_{\infty}(k)$ a Lyapunov function $V(\Delta x)=$ $\Delta x^{T} P \Delta x$ is generated. Additionally, an upper bound is derived such that $\max \left(A(k+i) \in \Omega, i \geq 0 J_{\infty}(k)\right) \leq V(\Delta x(k \mid k))$. The original optimization problem can be reformulated as:

$$
\min _{\Delta u(k+i \mid k), i=0,1, \ldots, \infty} V(\Delta x(k \mid k))
$$

which still implicitly depends on uncertainties. This problem leads to an optimization problem involving LMIs. Let $\Delta x(k)=\Delta x(k \mid k)$ be the state of the uncertain system (14) measured at sampling time $k$. State feedback matrix $F$ in the control law $\Delta u(k+i \mid k)=F \Delta x(k+i \mid k), i \geq 0$ minimizing the upper bound $V(\Delta x(k \mid k))$ is given by

$$
F=Y Q^{-1}
$$

where $Q$ and $Y$ are obtained from the solution of the following linear minimization problem, using LMIs:

$$
\begin{aligned}
\min _{\gamma, Q, Y} & \gamma \\
\text { s.t. } & {\left[\begin{array}{ccc}
1 & \Delta x(k \mid k)^{T} \\
\Delta x(k \mid k) & Q
\end{array}\right] \succeq 0, } \\
& {\left[\begin{array}{cccc}
Q & Q A_{j}^{T}+Y^{T} B^{T} & Q Q_{1}^{\frac{1}{2}} & Y^{T} R^{\frac{1}{2}} \\
A_{j} Q+B Y & Q & 0 & 0 \\
Q_{1}^{\frac{1}{2}} Q & 0 & \gamma I & 0 \\
R^{\frac{1}{2}} Y & 0 & 0 & \gamma I
\end{array}\right] \succeq 0, } \\
& j=1,2, \ldots, L
\end{aligned}
$$

The application of (19) represents a closed loop control which guarantees the robust stability of the system. An efficient solution algorithm for LMI problem (19) can be the SDP (Vandenberghe and Boyd, 1996).

\subsection{Constraints handling in minimax MPC}

As discussed in Section 2.1, the urban traffic system has several constraints which must be satisfied. The robust LMI based control offers input and output constraint handling as well. In terms of the invariant ellipsoids (Boyd et al., 1994) physical limitations can be incorporated into the robust MPC algorithm as sufficient LMI (Kothare et al., 1996) where the constraints are given in quadratic form. Thus, the system has to be subject to symmetric constraints of the following form:

$$
\underline{v} \leq v \leq \bar{v}
$$

where $|\underline{v}|=\bar{v}$. In our case the symmetric form (20) implies that the control input constraint has to be given as follows:

$$
\left|\Delta u_{z}(k+i \mid k)\right| \leq u_{z}^{*}(k), i \geq 0, z=1,2, \ldots, n_{u},
$$

Due to the symmetric definition $u_{z}^{*}(k)$ is defined conditionally. $u_{z}^{*}(k)=u_{z, \max }(k)-u_{z}^{N}$ if $u_{z}^{N} \geq u_{z, \max }(k) / 2$, else $u_{z}^{*}(k)=u_{z}^{N}$. Therefore, the original constraint (5) may turn more tight. Nevertheless, it does not reduce the control efficiency as urban traffic has a transient dynamics. For example, if link $z$ has very low nominal state $x_{z}^{N}$ with $u_{z}^{*}(k)=u_{z}^{N}$ potential $u_{z, \max }(k)$ is reduced. It may not be utilized at all by reason that the extreme increase of $x_{z}$ is not realistic from one time step $k$ to the next. At the same time the centering process (9) is continuously updated in a couple of time steps. Thus, $u_{z}^{*}(k)$ can follow the growth of $x_{z}$. Finally, the maximum green time constraints on each component of $\Delta u(k+i \mid k)$ can be expressed as an LMI according to the book of Boyd et al. (1994) and using Schur complement:

$$
\begin{aligned}
& {\left[\begin{array}{cc}
X & Y \\
Y^{T} & Q
\end{array}\right] \succeq 0, \text { with } X_{z z} \leq\left(u_{z}^{*}\right)^{2},} \\
& z=1,2, \ldots, n_{u}
\end{aligned}
$$

It has to be noted that using $u_{z}^{*}$ as constraint bound, zero green time may be calculated. Therefore, permanent red signal (in the next control interval) can be resulted which can be admissible depending of the concept of traffic management. At the same time, if permanent red is not allowed or there is a pedestrian stage in parallel, a non-zero minimum green time (according to the pedestrian stage) have to be ensured. This can be done easily such that the system automatically enlarges the lost time $L_{j}$ with the current pedestrian green time in the next cycle.

The linear combination constraints on green times (6) has to be guaranteed as well in SDP algorithm. Based on the deduction of (22) of Kothare et al. (1996) the LMI constraint of the sum of green times is derived (see App. A). Thus, the maximal value of the sum of green times at junction $j$ is formulated as below

$$
\left[\begin{array}{cc}
b_{j}^{2} & w_{j}^{T} Y \\
Y^{T} w_{j} & Q
\end{array}\right] \succeq 0, j=1,2, \ldots, J
$$

where $b_{j}$ is the maximal value allowed, $w_{j}^{T}$ the vector to allocate the current rows and columns of $Y$ according to 
$\Delta u$. Constraint $b_{j}$ depends on the nominal control inputs $u^{N}$ :

$$
b_{j}=T_{j, \max }-\sum_{z=1}^{O_{j}} u_{z}^{N}(k)
$$

It has to be noted that in case of less dense traffic the SDP algorithm does not necessarily result in all the possible green times $\left(T_{j, \max }\right)$. Nevertheless, according to the general concept of the traffic engineering (utilization of all available green times) it is worth distributing the rest proportionally among the stages. At the same time, these additional green times do not disturb the model (8) as the green time intervals are proportional to the number of exiting vehicles.

Furthermore, for better performance, additional constraints can be imposed on centered process output $\Delta y(k)$ (i.e. the states in our case). The use of the output constraints may contribute to avoiding spill-over phenomenon on links in the controlled traffic area applying the following LMI:

$$
\begin{aligned}
& {\left[\begin{array}{cc}
Q & Q A_{j}^{T}+Y^{T} B^{T} \\
A_{j} Q+B Y & Z
\end{array}\right] \succeq 0,} \\
& \text { with } Z_{z z} \leq \Delta y_{z, \text { max }}^{2}=\Delta x_{z, \text { max }}^{2}, \\
& z=1,2, \ldots, n_{y}, j=1,2, \ldots, L,
\end{aligned}
$$

\section{SIMULATION AND RESULTS}

The minimax MPC was verified in a closed loop simulation environment. We used microscopic traffic simulator (VISSIM) for traffic network modeling and the LMITOOL package of SCILAB (Nikoukhah et al., 1995) for the control process. By using simulations, we could compare nominal and robust control strategies in presence of uncertainties. The simulation is realized as online control. In each control cycle the measurements of the states are forwarded to SCILAB. After minimizing the cost function, the new green times return to the traffic simulator.

During the simulations, we used a fictional test network including 4 controlled intersections and 16 controlled links (states). The lengths of links alter between 100 and 250 meters. State uncertainties were involved in the control scheme determining the upper and lower bounds of unmeasured traffic flows. Control disturbances were not considered. Therefore, polytopic set (12) was applied. We applied centered traffic model with nominal control inputs by solving problem (9). Inflow demands $\left(d^{N}\right)$ were determined by real word data considering average traffic measurements in District 10 of Budapest during peak hours. We defined two uncertain cases for the simulations. One is with uncertain link 1 (see Fig. 3) and another with two uncertain locations (link 1 and 2 ).

We simulated three different scenarios with $\pm 10 \%, \pm 20 \%$ and $\pm 30 \%$ state uncertainties in both cases. The uncertainties were generated by non-controlled junctions as nonmeasurable vehicle flows. The dynamics of the disturbing fluctuations had normal distribution. To compare the effectiveness we simulated robust (RMPC) and nominal MPC (NMPC) strategies as well. The nominal MPC was applied with $L=1$ and $A=I$ since it did not take any uncertainties into consideration. Nevertheless, the same

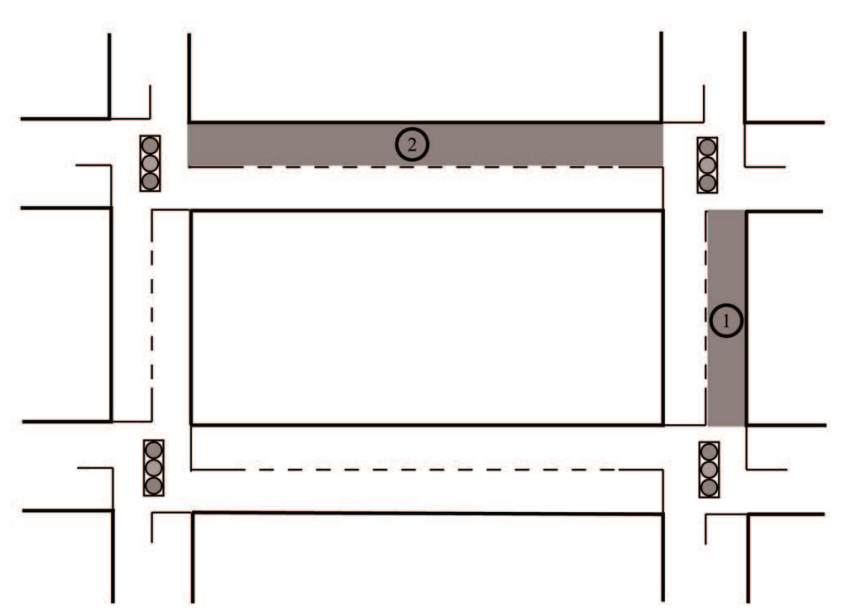

Fig. 3. Simulation traffic network with state uncertainties

SDP algorithm (19) was used for robust and nominal MPC with LMI constraints $(22,23,25)$. The simulation time was 1800 seconds. The cycle time was set to $90 \mathrm{sec}$.

Four traffic parameters were analyzed during the simulations. The first parameter is the Number of vehicles that have left the network which gives a general result considering the network. Average number of stops per vehicles is another illustrative parameter to show the dynamics of the traffic. The Average delay time per vehicle determines the mean time delay calculated from all vehicles observed in the network compared to the ideal travel time (no other vehicles, no signal control). Finally, the Average total travel time per vehicle was chosen to measure which is the average of all vehicles' travel times needed to pass the network. The results are displayed in Tables 1 and 2, respectively. The rows Change of Tables 1, 2 represent the variation of current traffic parameters compared to the nominal MPC strategies.

Table 1. Simulation results comparing the robust and nominal control strategies with one uncertainty

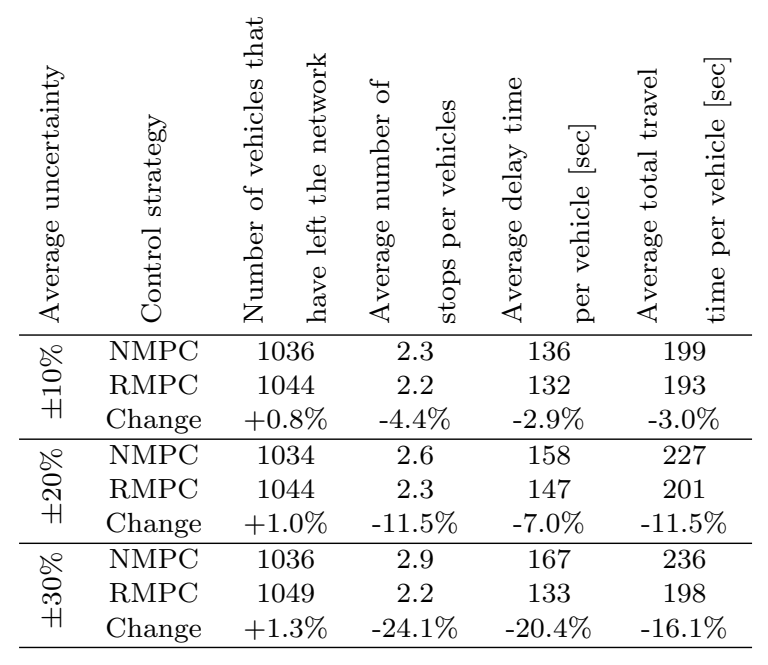

Table 1 and 2 shows that all traffic parameters changed in the right way. The measure of amelioration is similar in both cases. The robust property of the signal plan design improves the system performance in all important traffic parameters. We also can observe that the number of 
Table 2. Simulation results comparing the robust and nominal control strategies with two uncertainties

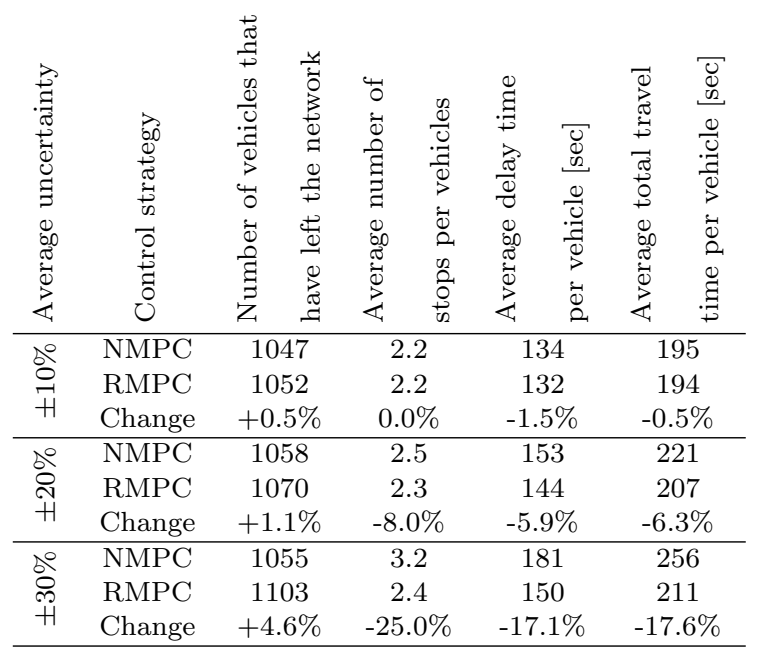

uncertainties in this test network did not necessary imply stronger disturbances regarding the whole traffic network.

\section{CONCLUSION}

The paper introduces an uncertainty modeling framework for control of urban traffic networks. Recognizing the need for robust control in urban traffic management, a constrained and robust model predictive algorithm is used to minimize traffic related objectives. The result of the robust predictive algorithm can be given as a convex set of LMI conditions. The closed-loop method is demonstrated via simulations.

Nevertheless, the authors intend to investigate the same problem in an alternative way. As a part of future research additive uncertainty approach and a different minimax MPC technique (introduced in Löfberg (2003)) are planned to be involved for robust traffic control.

\section{REFERENCES}

Aboudolas, K., Papageorgiou, M., and Kosmatopoulos, E. (2009). Store-and-forward based methods for the signal control problem in large-scale congested urban road networks. Transportation Research Part C: Emerging Technologies, 17, 163-174. doi:10.1016/j.trc.2008.10.002.

Boyd, S., El Ghaoui, L., Feron, E., and Balakrishnan, V. (1994). Linear Matrix Inequalities in System and Control Theory. SIAM Studies in Applied Mathematics. SIAM.

de Oliveira, L. and Camponogara, E. (2010). Multiagent model predictive control of signaling split in urban traffic networks. Transportation Research Part C: Emerging Technologies, 18(1), 120-139. doi: 10.1016/j.trc.2009.04.022. Information/Communication Technologies and Travel Behaviour; Agents in Traffic and Transportation.

Diakaki, C., Papageorgiou, M., and McLean, T. (1999). Application and evaluation of the integrated trafficresponsive urban corridor control strategy in-tuc in glasgow. In CD-ROM of the 78th Annual Meeting of the Transportation Research Board, 990310. Washington, D.C., U.S.A.
Gazis, D. and Potts, R. (1963). The oversaturated intersection. In: Proceedings of the Second International Symposium on Traffic Theory, London, UK, 221-237.

Kothare, M., Balakrishnan, V., and Morari, M. (1996). Robust constrained model predictive control using linear matrix inequalities. Automatica, 32(10), 1361-1379.

Kulcsár, B., Bécsi, T., and Varga, I. (2005). Estimation of dynamic origin destination matrix of traffic systems. Periodica Polytechnica ser. Transp. Eng, 33(1-2), 3-14.

Löfberg, J. (2003). Minimax approaches to robust model predictive control. Ph.D. thesis, Linköping University, Linköping, Sweden.

Nikoukhah, R., Delebecque, F., and El Ghaoui, L. (1995). Lmitool: a package for lmi optimization in scilab, user's guide. Technical report, INRIA.

Rawlings, J.B. and Muske, K.R. (1993). The stability of constrained receding horizon control. IEEE Trans. Autom. Control, 38, 1512-1516. doi:10.1109/9.241565.

Tettamanti, T., Varga, I., Kulcsár, B., and Bokor, J. (2008). Model predictive control in urban traffic network management. In CD ISBN:978-1-4244-2505-1, 15381543. Ajaccio, Corsica, France.

Vandenberghe, L. and Boyd, S. (1996). Semidefinite programming, volume 38. SIAM Review.

Vigos, G., Papageorgiou, M., and Wang, Y. (2007). Real-time estimation of vehicle-count within signalized links. Transportation Research Part C. doi: 10.1016/j.trc.2007.06.002.

Welch, G. and Bishop, G. (1995). An introduction to the kalman filter. Technical Report TR95-041, University of North Carolina at Chapel Hill.

Zafiriou, E. (1990). Robust model predictive control of processes with hard constraints. Computers and Chemical Engineering, 14, 359-371. doi:10.1016/00981354(90)87012-E.

\section{Appendix A. DERIVATION OF THE LINEAR COMBINATION CONSTRAINTS ON GREEN TIMES}

Let $\epsilon=\left\{\Delta x \mid \Delta x^{T} Q^{-1} \Delta x \leq 1\right\}=\left\{\Delta x \mid \Delta x^{T} P \Delta x \leq \gamma\right\}$ an invariant ellipsoid for the predicted states of the uncertain system. At sampling time $k$ consider the Euclidean norm constraint for junction $j$ :

$$
\left\|w_{j}^{T} \Delta u(k+i \mid k)\right\|_{2} \leq b_{j}, i \geq 0
$$

Following Boyd et al. (1994), we can formulate the next expression:

$$
\begin{aligned}
& \max _{i \geq 0}\left\|w_{j}^{T} \Delta u(k+i \mid k)\right\|_{2}^{2}= \\
& \max _{i \geq 0}\left\|w_{j}^{T} Y Q^{-1} \Delta x(k+i \mid k)\right\|_{2}^{2} \leq \\
& \max _{\Delta x \in \epsilon}\left\|w_{j}^{T} Y Q^{-1} \Delta x\right\|_{2}^{2}= \\
& \lambda_{\max }\left(w_{j}^{T} Q^{-\frac{1}{2}} Y^{T} Y Q^{-\frac{1}{2}} w_{j}\right)
\end{aligned}
$$

The last inequality is a generalized eigenvalue problem and it holds true at all times $i \geq 0$ if the following LMI holds:

$$
\left[\begin{array}{cc}
b_{j}^{2} & w_{j}^{T} Y \\
Y^{T} w_{j} & Q
\end{array}\right] \succeq 0
$$

